\title{
Filter Design for a Compressive Sensing Delay and Doppler Estimation Framework
}

\author{
Misagh Khayambashi, A. Lee Swindlehurst \\ Department of EECS, University of California, Irvine \\ Irvine, CA 92697, USA \\ \{mkhayamb, swindle\}@uci.edu
}

\begin{abstract}
The theory of compressive sensing (CS) aims to find efficient signal acquisition and recovery techniques with the aid of prior knowledge about the signal. While traditionally applied to sparse vectors, CS has been extended to analog signals with more general structures. The use of $\mathrm{CS}$ in delay and Doppler estimation in radar application has recently received attention from the signal processing community. In this paper, we adopt one of the available CS frameworks for delay and Doppler estimation and optimize the deployed filter in this framework. The optimization criterion is the Bayesian Crámer Rao Bound of delay estimation and Doppler in general additive Gaussian interference. An iterative algorithm is proposed to solve the optimization problem and the results are compared with the prototype filter design available in the literature.
\end{abstract}

\section{INTRODUCTION}

The theory of compressive sensing deals with finding efficient measurement-recovery pairs given some prior information on the structure of the object to be measured and recovered [1], [2], [3]. The efficiency of the measurement scheme is usually interpreted as minimizing the required effort to extract sufficient information about the object, where sufficiency translates into recoverability of the object from gathered information through some recovery algorithm. On the other hand, the efficiency of recovery algorithm is usually assessed by its computational complexity.

The traditional theory of compressive sensing focuses on measuring sparse vectors, where the object to be measured is a finite length vector and the prior information is the sparsity of this vector. Motivated by the practical advantages of compressive sensing, similar frameworks have been proposed to deal with continuous signals and various other mathematical objects. Furthermore, the prior structure has been extended from sparsity to more general priors [4].

A promising application for compressive sensing framework is delay-Doppler estimation in radar systems [5], [7], [8]. In conventional radar systems, the common approach can be thought of as a search over all possible candidates values of delay and Doppler. However, the availability of prior information on the delay and Doppler characteristics of the scanned scene, such as information about the number of targets, can potentially simplify the implementation of the system by smart incorporation of prior information into the measurement/recovery procedure.

Integration of compressive sensing ideas and the delayDoppler estimation problem has been studied from various perspectives in the literature [5], [6], [7], [8].
In [9], a specific class of periodic signals are used for probing the environment and the identifiability of target(s) delay and Doppler from the received echo is examined. For the problem to be identifiable, it is shown that the probing signal and the front-end filter used in the proposed structure should satisfy certain conditions in frequency domain. Furthermore, a measurement/recovery method is adopted from [10] and the performance of estimation versus SNR of AWGN is evaluated through simulation. Interestingly, The identifiability conditions obtained in [9] do not specify a unique signal/filter choice. Rather, any signal/filter pair satisfying the identifiability conditions can serve the purpose. Consequently, one may wonder if it is possible to define a proper optimality criterion and find the "best" signal/filter pair. This article attempts to find such an optimum filter/signal pair.

A well-established method for evaluating the performance of any unbiased estimation framework is to compare its error performance with the best possible error performance, namely the Crámer Rao bound (CRB) . In addition, it is a well-known fact that the interference in radar applications can deviate significantly from AWGN model and that incorrect assumptions about interference model can significantly degrade estimation/detection performance [11], [12], [13], [13]. As a result, this article takes the CRB in general Gaussian interference as the optimzality criterion for finding the best signal/filter pair. Although not capable of modeling all empirical interference data, Gaussian processes can model a wide range of realworld interferences due to the flexibility in rhe choice of their covariance function.

The rest of this article is organized as follows. In Section II, the framework of [9] is reviewed. A brief review of Bayesian Crámer Rao bound is provided in Section III .Section IV formulates the optimization problem in terms of an analog filter and discusses various choices for solving the optimization problem. An iterative algorithm is proposed to solve the optimization problem. Finally, Section V compares the BCRB performance of the 'optimum' filter and the prototype filter. A summary of the paper is provided in Section VI.

\section{Estimation OF DELAY AND DOPPLER}

Consider a radar system probing the environment with following signal [9] :

$$
x(t)=\sum_{n=0}^{N-1} x_{n} g(t-n T)
$$


where $g(t)$ is a unit energy pulse of bandwidth $\mathcal{W}$ and (essentially) temporally limited to $[0, T]$, and $x_{n} \in \mathbb{C}$. Note that the duration of $x(t)$ is $\mathcal{T}=N T$. Suppose that the received signal $r(t)$ consists of $K$ scaled, delayed, and Doppler-shifted echos of the transmitted signal, with each each corresponding to a scale-delay-Doppler triplet $\left(\alpha_{k}, \tau_{k}, \nu_{k}\right), k=1, \cdots, K$ :

$r(t)=\sum_{k=1}^{K} \alpha_{k} x\left(t-\tau_{k}\right) e^{j 2 \pi \nu_{k} t}=\sum_{i=1}^{K_{\tau}} \sum_{j=1}^{K_{i, \nu}} \alpha_{i j} x\left(t-\tau_{i}\right) e^{j 2 \pi \nu_{i j} t}$

where the second equality has been rewritten assuming that there are $K_{\tau} \leq K$ distinct delays, and that for the $i$ th distinct delay there are $K_{i, \nu}$ distinct Doppler shifts. The question is whether the set of delays and Dopplers is identifiable from $r(t)$.

It is shown in [9] that under the following assumptions
1) $\left(\tau_{i}, \nu_{i j}\right) \in\left[0, \tau_{\max }\right] \times\left[-\nu_{\max } / 2, \nu_{\max } / 2\right]$
2) $\tau_{\max }<T$
3) $\nu_{\max } \ll \mathcal{W}$
4) all $x_{n}>0$

the delay and Doppler parameters are identifiable as long as $\mathcal{T W} \geq 8 \pi K_{\tau} K_{\nu, \max }$, where $K_{\nu, \max }=\max _{i} K_{i, \nu}$.

In order to recover delay and Dopplers, $r(t)$ is rewritten as:

$$
\begin{aligned}
r(t) & =\sum_{i=1}^{K_{\tau}} \sum_{n=0}^{N-1} a_{i}[n] g\left(t-\tau_{i}-n T\right) \\
a_{i}[n] & =\sum_{j=1}^{K_{\nu, i}} \alpha_{i j} x_{n} e^{j 2 \pi \nu_{i j} n T}
\end{aligned}
$$

Reference [9] deploys a variation of the recovery method in [10]. In [9], $r(t)$ is first passed through an LPF with response $s^{*}(-t)$, where $s^{*}(-t)$ is limited to frequency band $\left[-\frac{\pi}{T} p, \frac{\pi}{T} p\right]$ and $p$ is an even number satisfying $p \geq 2 K_{\tau}$. Then, the output of filter is uniformly sampled at multiples of $T / p$. These samples are then processed through a digital filter bank, and the output of the filter bank is shown to have a connection with the unknown time delays. For the filter bank to exist, it is required that the spectrum of $g(t)$ and $s(t)$ are non-zero over the bandwidth $\left[-\frac{\pi}{T} p, \frac{\pi}{T} p\right]$. The time delays are estimated from the output of filter bank by ESPIRIT algorithm [15]. Once the delays are known, subspace methods for estimation can be used to estimate the unknown Dopplers and attenuations. The reader is referred to [9] for more details.

\section{BAYESIAN CRB: APPLICATION OF MULTIVARIATE VAN TREES INEQUALITY}

Consider the following noisy received signal:

$$
\begin{aligned}
& r(t)=\sum_{i=1}^{K_{\tau}} \sum_{n=0}^{N-1} a_{i}[n] g\left(t-\tau_{i}-n T\right)+w(t) \\
& a_{i}[n] \triangleq \sum_{j=1}^{K_{\nu, i}} \alpha_{i j} x_{n} e^{j 2 \pi \nu_{i j} n T}
\end{aligned}
$$

where the interference $w(t)$ is a complex Gaussian process with iid real and imaginary components:

$$
\Re\{w(t)\}, \Im\{w(t)\} \sim \mathcal{G P}\left(0, C_{w}\left(t, t^{\prime}\right)\right)
$$

The received noisy signal $r(t)$ goes through an analog real filter $s(t)$, generating the signal $q(t)$ :

$$
\begin{aligned}
& q(t)=v(t)+u(t) \\
& v(t) \triangleq \sum_{i=1}^{K_{\tau}} \sum_{n=0}^{N-1} a_{i}[n] h\left(t-\tau_{i}-n T\right) \\
& h(t) \triangleq g(t) * s(t) \\
& u(t)=w(t) * s(t)
\end{aligned}
$$

and $u(t)$ is a complex Gaussian process with iid real and imaginary components::

$$
\begin{aligned}
& \Re\{w(t)\}, \Im\{w(t)\} \sim \mathcal{G P}\left(0, C_{u}\left(t, t^{\prime}\right)\right) \\
& C_{u}\left(t, t^{\prime}\right)=\iint d \tau d \tau^{\prime} s(t-\tau) s\left(t^{\prime}-\tau^{\prime}\right) C_{w}\left(\tau, \tau^{\prime}\right)
\end{aligned}
$$

The filtered signal $q(t)$ is sampled every $T / p$ seconds. The sample vector is:

$$
\begin{aligned}
\mathbf{q} & \triangleq[q(T / p), q(2 T / p), \cdots, q(T)]^{T} \\
& =[v(T / p), v(2 T / p), \cdots, v(T)]^{T}+ \\
& {[u(T / p), u(2 T / p), \cdots, u(T)]^{T} } \\
& \triangleq \mathbf{v}+\mathbf{u}
\end{aligned}
$$

Also, $\mathbf{u}$ is a complex multivariate Gaussian with iid real and imaginary components:

$$
\begin{aligned}
\Re\{\mathbf{u}\}, \Im\{\mathbf{u}\} & \sim \mathcal{G}\left(0, \boldsymbol{\Sigma}_{\mathbf{U}}\right) \\
{\left[\boldsymbol{\Sigma}_{\mathbf{U}}\right]_{i, j} } & =C_{u}(i T / p, j T / p)
\end{aligned}
$$

Note that both the deterministic vector $\mathbf{v}$ and the covariance of multivariate $\mathbf{u}$ are vector-valued functionals of $s(t)$. The goal is to find the BCRB (Bayesian Crámer Rao Bound) of estimate of parameter vector $\boldsymbol{\theta}$ from $\mathbf{q}$ and then choose the filter $s(t)$ that minimizes BCRB. The parameter vector $\boldsymbol{\theta}$ consists of the unknown delays $\left\{\tau_{i}, i=1, \cdots, K_{\tau}\right\}$, Doppler shifts $\left\{\nu_{i j}, i=1, \cdots, K_{\tau}, j=1, \cdots, K_{\nu, i}\right\}$, and attenuations $\left\{\alpha_{i j}, i=1, \cdots, K_{\tau}, j=1, \cdots, K_{\nu, i}\right\}$ :

$$
\begin{aligned}
\boldsymbol{\theta}= & {\left[\boldsymbol{\kappa}_{1}^{T}, \cdots, \boldsymbol{\kappa}_{K_{\tau}}^{T}\right]^{T} } \\
\boldsymbol{\kappa}_{i} \triangleq & {\left[\tau_{i}, \nu_{i, 1}, \cdots, \nu_{i, K_{\nu, i}}, \alpha_{i, 1}, \cdots, \alpha_{i, K_{\nu, i}}\right]^{T} } \\
& i \in\left\{1, \cdots, K_{\tau}\right\}
\end{aligned}
$$

A simplified version of Van Trees inequality [16] can be used to extend the concept of CRB to Bayesian CRB. Specifically, given a prior distribution on $\boldsymbol{\theta}, \mathcal{P}_{\Theta}(\boldsymbol{\theta})$, a posterior distribution on $\mathbf{q}$ given $\boldsymbol{\theta}, \mathcal{P}_{\mathbf{Q} \mid \Theta}(\mathbf{q}, \boldsymbol{\theta})$, and any positive definite matrix $\mathbf{B}$, the following inequality holds for any unbiased estimator $\hat{\boldsymbol{\theta}}(\mathbf{q})$ of $\boldsymbol{\theta}$ :

$$
\begin{aligned}
& \int d \boldsymbol{\theta} \mathcal{P}_{\boldsymbol{\Theta}}(\boldsymbol{\theta}) \mathbf{E}_{\mathbf{Q} \mid \boldsymbol{\Theta}}\left\{[\hat{\boldsymbol{\theta}}(\mathbf{q})-\boldsymbol{\theta}]^{T} \mathbf{B}^{-1}[\hat{\boldsymbol{\theta}}(\mathbf{q})-\boldsymbol{\theta}]\right\} \\
& \geq \frac{1}{\mathcal{I}+\int \operatorname{tr}\left\{\mathbf{B}^{T} \mathbf{I}(\boldsymbol{\theta})\right\} \mathcal{P}_{\boldsymbol{\Theta}}(\boldsymbol{\theta}) d \boldsymbol{\theta}}
\end{aligned}
$$


where $\mathbf{I}(\boldsymbol{\theta})$ is the Fisher information matrix, $\mathbf{E}$ denotes expectation, and $\mathcal{I}$ is a term that does not depend on $s(t)$ :

$$
\mathcal{I}=\int d \boldsymbol{\theta} \frac{1}{\mathcal{P}_{\boldsymbol{\Theta}}(\boldsymbol{\theta})} \sum_{i=1}^{M} \sum_{j=1}^{M}[\mathbf{B}]_{i j} \frac{\partial \mathcal{P}_{\boldsymbol{\Theta}}(\boldsymbol{\theta})}{\partial \theta_{i}} \frac{\partial \mathcal{P}_{\boldsymbol{\Theta}}(\boldsymbol{\theta})}{\partial \theta_{j}}
$$

Finally, we can choose $\mathbf{B}^{-1}$ to be a positive diagonal weighting matrix:

$$
\mathbf{B}^{-1}=\operatorname{diag}\left\{b_{1}^{-1}, \cdots, b_{p}^{-1}\right\}, b_{i}>0
$$

With this choice, the elements of $\mathbf{B}$ can be used for two purposes. First, it is possible to bring the numerical values of different parameters of different dimensions (for example, delay measured in $\mu s$ but Doppler shift measured in $k H z$ ) in the same range in order for all parameters to have a fair contribution to BCRB. Second, different weights can be assigned to different parameters depending on their importance.

Since $\mathbf{B}$ is a diagonal matrix, only the diagonal elements of I contribute to the bound in Equation 22. It can be shown that:

$$
\mathbf{I}_{m m}=2 \sum_{i=1}^{p} \sum_{j=1}^{p}\left[\boldsymbol{\Sigma}_{\mathbf{U}}^{-1}\right]_{i j} \frac{\partial v_{i}^{*}}{\partial \theta_{m}} \frac{\partial v_{j}}{\partial \theta_{m}}
$$

where $v_{i}$ is the $i$ th element of vector $\mathbf{v}$. Plugging back into Equation 22, the component of lower bound that depends on $s(t)$ is equal to:

$$
\begin{aligned}
& \int \operatorname{tr}\left\{\mathbf{B}^{T} \mathbf{I}(\boldsymbol{\theta})\right\} \mathcal{P}_{\boldsymbol{\Theta}}(\boldsymbol{\theta}) d \boldsymbol{\theta}=\sum_{m=1}^{M} \int \mathbf{I}_{m m} b_{m} \mathcal{P}_{\boldsymbol{\Theta}}(\boldsymbol{\theta}) d \boldsymbol{\theta} \\
& =\sum_{m=1}^{M} \int\left(2 \sum_{i=1}^{p} \sum_{j=1}^{p}\left[\boldsymbol{\Sigma}_{\mathbf{U}}^{-1}\right]_{i j} \frac{\partial v_{i}^{*}}{\partial \theta_{m}} \frac{\partial v_{j}}{\partial \theta_{m}}\right) b_{m} \mathcal{P}_{\boldsymbol{\Theta}}(\boldsymbol{\theta}) d \boldsymbol{\theta} \\
& =2 \sum_{i=1}^{p} \sum_{j=1}^{p}\left[\boldsymbol{\Sigma}_{\mathbf{U}}^{-1}\right]_{i j} \sum_{m=1}^{M} b_{m} \int \mathcal{P}_{\boldsymbol{\Theta}}(\boldsymbol{\theta}) d \boldsymbol{\theta} \frac{\partial v_{i}^{*}}{\partial \theta_{m}} \frac{\partial v_{j}}{\partial \theta_{m}} \\
& =2\left\|\boldsymbol{\Sigma}_{\mathbf{U}}^{-1} \odot \mathbf{V}\right\|_{1}
\end{aligned}
$$

where $\|\cdot\|_{1}$ denotes the summation of elements of matrix, $\odot$ is Hadamard product, and $\mathbf{V}$ is a $p \times p$ matrix defined by:

$$
[\mathbf{V}]_{i j}=\sum_{m=1}^{M} b_{m} \int \mathcal{P}_{\boldsymbol{\Theta}}(\boldsymbol{\theta}) d \boldsymbol{\theta} \frac{\partial v_{i}^{*}}{\partial \theta_{m}} \frac{\partial v_{j}}{\partial \theta_{m}}
$$

As mentioned earlier, the lower bound of Equation 22 is to be minimized over the propoerly constrained space of filters. This optimization problem is equivalent to maximizing $\left\|\boldsymbol{\Sigma}_{\mathbf{U}}^{-1} \odot \mathbf{V}\right\|_{1}$ :

$$
s^{\mathrm{opt}}(t)=\arg \max _{s(t)}\left(\left\|\boldsymbol{\Sigma}_{\mathbf{U}}^{-1} \odot \mathbf{V}\right\|_{1}\right)
$$

subject to energy and identifiability constraint. In the following sections, we consider the general and simplified versions of this optimization problem and evaluate the performace of resulting filter with the performance of prototype filter available in the literature.

\section{Filter Optimization}

The proposed optimization problem seems analytically intractable for various reasons. Apart from the complicated interaction between cost function and the optimization variable $s(t)$, the simultaneous presence of matrix inversion in $\boldsymbol{\Sigma}_{\mathbf{U}}^{-1}$ and dependence of $\boldsymbol{\Sigma}_{\mathbf{U}}$ on $s(t)$ discourages a direct analytical approach toward solving the optimization problem.

If numerical methods are to be used for optimization, it is preferrable to prove the convexity of the problem and exploit the advantages of convex optimization. In the proposed optimization problem, even if the problem can be shown to be convex, a fixed size discretization of the filter should be fed into the optimization algorithm. Since the temporal support of the optimum filter is not known a priori, the use of conventional optimization algorithms may lead to sub-optimal solutions. However, if an estimate of the temporal bounds of optimal filter is available, conventional optimization algorithms with convergence and monotonicity guarantees can be used.

In order to partially circumvent the mentioned drawbacks, we propose an iterative algorithm based on the Lagrangian formulation of the optimization problem.

The Lagrangian with energy constraint is:

$$
\mathcal{L}(s(t), \lambda)=\left\|\boldsymbol{\Sigma}_{\mathbf{U}}^{-1} \odot \mathbf{V}\right\|_{1}+\lambda\left(\int s^{2}(t) d t-1\right)
$$

As a necessary condition for optimality of $s(t)$, the functional derivative of $\mathcal{L}$ w.r.t $s(t)$ should equal the 0 function. From functional derivative theory:

$$
\frac{\partial \mathcal{L}}{\partial s}(t)=\left\|\frac{\partial \boldsymbol{\Sigma}_{\mathbf{U}}^{-1}}{\partial s}(t) \odot \mathbf{V}+\boldsymbol{\Sigma}_{\mathbf{U}}^{-1} \odot \frac{\partial \mathbf{V}}{\partial s}(t)\right\|_{1}+2 \lambda s(t)
$$

with:

$$
\begin{aligned}
& {\left[\frac{\partial \mathbf{V}}{\partial s}(t)\right]_{i j}=\sum_{m=1}^{M} b_{m} \times} \\
& \int \mathcal{P}_{\boldsymbol{\Theta}}(\boldsymbol{\theta}) d \boldsymbol{\theta}\left\{\frac{\partial v_{i,\left(\theta_{m}\right)}^{*}}{\partial s}(t) v_{j,\left(\theta_{m}\right)}+v_{i,\left(\theta_{m}\right)}^{*} \frac{\partial v_{j,\left(\theta_{m}\right)}}{\partial s}(t)\right\}
\end{aligned}
$$

where $v_{j,\left(\theta_{m}\right)} \triangleq \partial v_{j} / \partial \theta_{m}$. It can be readily shown that:

$$
\begin{aligned}
v_{y,\left(\tau_{z}\right)}= & -\sum_{n=0}^{N-1} a_{z}[n] \dot{h}\left(y \frac{T}{p}-\tau_{z}-n T\right) \\
v_{y,\left(\nu_{z w}\right)}= & \sum_{n=0}^{N-1} \alpha_{z w} x_{n}(j 2 \pi n T) e^{j 2 \pi \nu_{z w} n T} \times \\
& h\left(y \frac{T}{p}-\tau_{z}-n T\right) \\
v_{y,\left(\alpha_{z w}\right)}= & \sum_{n=0}^{N-1} x_{n} e^{j 2 \pi \nu_{z w} n T} h\left(y \frac{T}{p}-\tau_{z}-n T\right)
\end{aligned}
$$

Also, using functional calculus:

$$
\frac{\partial v_{y,\left(\tau_{z}\right)}}{\partial s}(t)=-\sum_{n=0}^{N-1} a_{z}[n] \dot{x}\left(y \frac{T}{p}-\tau_{z}-n T-t\right)
$$




$$
\begin{aligned}
& \frac{\partial v_{y,\left(\nu_{z w}\right)}}{\partial s}(t)= \sum_{n=0}^{N-1} \alpha_{z w} x_{n}(j 2 \pi n T) e^{j 2 \pi \nu_{z w} n T} \times \\
& x\left(y \frac{T}{p}-\tau_{z}-n T-t\right) \\
& \frac{\partial v_{y,\left(\alpha_{z w}\right)}}{\partial s}(t)=\sum_{n=0}^{N-1} x_{n} e^{j 2 \pi \nu_{z w} n T} x\left(y \frac{T}{p}-\tau_{z}-n T-t\right)
\end{aligned}
$$

Furthermore, the term $\frac{\partial \boldsymbol{\Sigma}_{\mathbf{U}}^{-1}}{\partial s}(t)$ can be simplified by using the identity:

$$
\frac{\partial\left(\boldsymbol{\Sigma}_{\mathbf{U}}^{-1}\right)}{\partial s}(t)=-\boldsymbol{\Sigma}_{\mathbf{U}}^{-1} \frac{\partial \boldsymbol{\Sigma}_{\mathbf{U}}}{\partial s}(t) \boldsymbol{\Sigma}_{\mathbf{U}}^{-1}
$$

with

$$
\begin{aligned}
{\left[\frac{\partial \boldsymbol{\Sigma}_{\mathbf{U}}}{\partial s}(t)\right]_{i j}=} & \int\left[C_{w}(\alpha, j T / p-t) s(i T / p-\alpha)\right. \\
& \left.+C_{w}(i T / p-t, \alpha) s(j T / p-\alpha)\right] d \alpha
\end{aligned}
$$

Now, in order to set Equation 30 to 0 and find a potentially optimum filter, the following equation has to be solved for $s(t)$ :

$$
\left\|\frac{\partial \boldsymbol{\Sigma}_{\mathbf{U}}^{-1}}{\partial s}(t) \odot \mathbf{V}+\boldsymbol{\Sigma}_{\mathbf{U}}^{-1} \odot \frac{\partial \mathbf{V}}{\partial s}(t)\right\|_{1}+2 \lambda s(t)=0
$$

Then, if $s_{0}(t, \lambda)$ is a solution of this equation, $\lambda$ is determined by applying the energy constraint to $s_{0}(t, \lambda)$. Due to the complicated dependence of $\boldsymbol{\Sigma}_{\mathbf{U}}^{-1}$ on $s(t)$, a direct analytical approach toward solving this equation seems futile. However, the resemblence between this problem and the problem of solving $f(x)=x$ for $x$ suggests that the same iterative methods used for solving the latter can be used.

It is common to solve $f(x)=x$ iteratively by plugging the $i$ th iterative solution $z^{(i)}$ into $f(z)$ and update the solution as $z^{(i+1)}=f\left(z^{(i)}\right)$. The iteration continues until some convergence criterion is satisfied. In a similar manner, we propose the following iterative procedure to solve Equation 40 for $s(t)$ :

$$
\begin{aligned}
& \hat{s}^{(i+1)}(t)=\left.\left\|\frac{\partial \boldsymbol{\Sigma}_{\mathbf{U}}^{-1}}{\partial s}(t) \odot \mathbf{V}+\boldsymbol{\Sigma}_{\mathbf{U}}^{-1} \odot \frac{\partial \mathbf{V}}{\partial s}(t)\right\|_{1}\right|_{s^{(i)}(t)} \\
& s^{(i+1)}(t)=\frac{\hat{s}^{(i+1)}(t)}{\sqrt{\int\left(\hat{s}^{(i+1)}(t)\right)^{2} d t}}
\end{aligned}
$$

where the second step is added to force the energy constraint.

In this article, no guarantee on monotonicity and global/local convergence of the proposed algorithm is provided. Rather, the goal is to compare both the BCRB and error performance of the resulting filter with the performance of the prototype filter of [9].

\section{Performance Evaluation}

In order to examine the CRB performance of the algorithm, the prototype design of [9] is used as the starting point of the proposed iterative algorithm. For each value of $p$, the pulse $g(t)$ is designed to be a properly truncated and normalized sinc, where the sinc is the inverse Fourier transform of

$$
\hat{G}_{0}(\omega)= \begin{cases}e^{-j \omega T / 2} & |\omega|<\pi \frac{p}{T} \\ 0 & \text { Otherwise }\end{cases}
$$

which means:

$$
g(t)=K \frac{\sin \left(\pi \frac{p}{T}(t-T / 2)\right)}{\pi \frac{p}{T}(t-T / 2)}[u(t)-u(t-T)]
$$

where the constant $K$ is determined by $\int g^{2}(t) d t=1$ and $u(t)$ is the unit step function. Furthermore, the initial filter is matched to $g(t)$. In order to choose $N$, we use the condition $\mathcal{T W} \geq 8 \pi K_{\tau} K_{\nu, \max }$. Writing $p$ as $p=2 K_{\tau}+2 R$ with $R \in$ $\mathbb{N} \cup\{0\}$, and taking $\mathcal{W}=\pi p / T$ and $K_{\nu, \max }=1$, the condition on $N$ is:

$$
N \geq \frac{4 K_{\tau}}{K_{\tau}+R}
$$

Also, the sequence $\left\{x_{n}\right\}$ is a random binary $( \pm 1)$ sequence.

The Gaussian process is assumed to follow an exponential model:

$$
C_{w}\left(t, t^{\prime}\right)=\xi^{-1}\left(\max _{t}|g(t)|^{2}\right) \exp \left(-\left(\frac{t-t^{\prime}}{T / p}\right)^{2}\right)
$$

corresponding to a correlation time of $T / p$. The positive scalar $\xi$ is a measure of SNR defined as the ratio of maximum instantaneous power of the pulse $g(t)$ to the expected instantaneous power of interference. $\xi$ is set to 1 for simulation. Finally, $\mathcal{P}(\tau)$ is assumed to be a uniform distribution over the interval $[0, T]$, and $T$ is set to $10 \mu \mathrm{s}$. Also note that the choice of the interference model and $g(t)$ can be arbitrary. Our choice is only for the purpose of illustration.

In the first scenario, there is a single target whose delay is uniformly distributed over [0 T], Doppler is uniformly spread over $.01 *[-p / 2 T p / 2 T] H z$, and reflection strength is equal to 1. For the purpose of illustration, the diagonal of $\mathbf{B}$ is adjusted to give all the significance to delay estimation (although any combination can be used). Figure 1 shows the variation of Bayesian Standard Deviation (BSD), i.e. $\sqrt{\mathrm{BCRB}}$, for different choices of $p$. The bold line belongs to the results obtained from matched filtering, while the dashed line represents the results obtained from the optimum filter. Clearly, the performance gain is considerable. Notably, the gain is more considerable in low$p$ regime which is of primary interest to compressive sensing strategies.

In the second scenario, two targets are considered. The delay distribution of first target is Gaussian centered at $T / 4$ with a spread of $T / 10$. For the second target, the distribution is centered at $T / 5$ with the same spread of $T / 10$. Note that the separation of centers is about the same as the spreads, a situation that causes ambiguity in target discrimination. Figure 2 depicts the BSD of first target's delay estimate for different choices of $p$. As is evident from the figure, the introduction of 'optimum' filter has significantly improved the target-discrimination capability of the estimator.

It is noteworthy that the authors have applied the same procedure to different scenarios. For example, $g(t)$ can be a chirp or other well know signals, the interference can be any complex model including periodically correlated interference, 


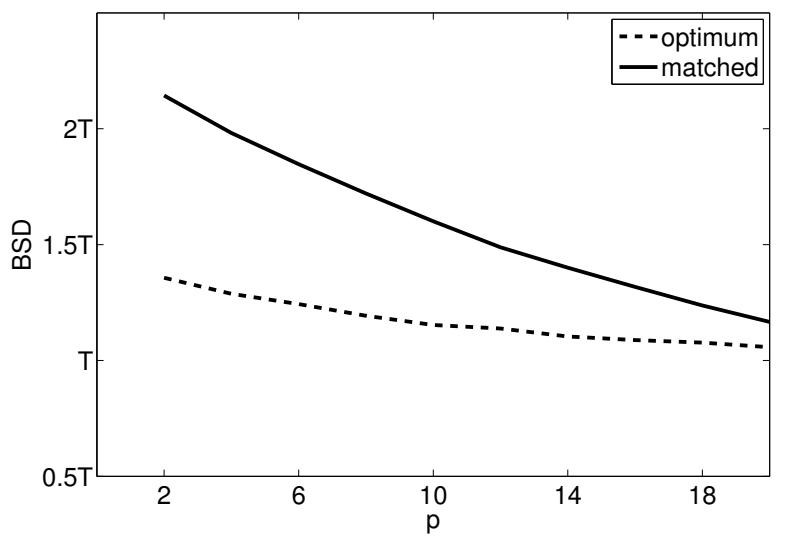

Fig. 1: Single target scenario: BSD vs. $p$ for prototype and 'optimal' filter

multiple targets with arbitrary parameter distributions can be introduced, and the weighting matrix $\mathbf{B}$ can be adjusted according to some prespecified requirement. Fortunately, the use of 'optimum' filter results in significant performance gain for all realistic scenarios, especially at low- $p$ regime.

\section{CONCLUSiON}

The compressive sensing framework of [9] for delay and Doppler estimation is adopted in this paper. After reviewing the mechanics of this framework, the degrees of freedom in the choice of front-end filter is used to optimized the performance. Specifically, the performance criterion is the Bayesian CRB of delay/Doppler estimation in presence of general Gaussian interference. An optimization problem is formulated in terms of the analog filter, and the 'optimum' analog filter is calculated by a fast iterative algorithm. While no proof of monotonicity or global/local convergence of the iterative algorithm is provided, the performance of the resulting filter is compared against the existing prototype filter design. The resulting filter boasts a significant performance gain compared to the available filter design. It is anticipated that the resulting filter outperforms the prototype filter in terms of statistical estimation error metrics too.

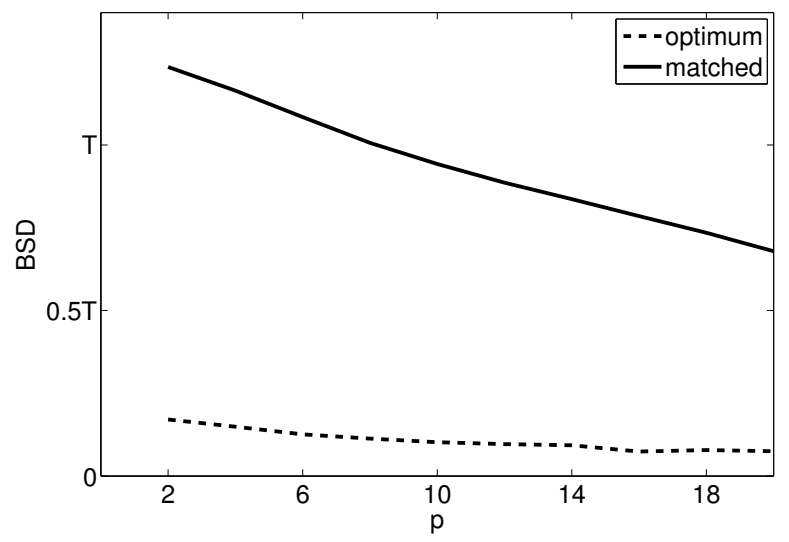

Fig. 2: Double target scenario: BSD vs. $p$ for prototype and 'optimal' filter

\section{REFERENCES}

[1] Cands, Emmanuel J. "Compressive sampling." Proceedings of the international congress of mathematicians. Vol. 3. 2006

[2] Baraniuk, Richard. "Compressive sensing." IEEE signal processing magazine 24.4 (2007).

[3] Cands, Emmanuel J., and Michael B. Wakin. "An introduction to compressive sampling." Signal Processing Magazine, IEEE 25.2 (2008): 21-30.

[4] Duarte, Marco F., and Yonina C. Eldar. "Structured compressed sensing: From theory to applications." Signal Processing, IEEE Transactions on 59.9 (2011): 4053-4085.

[5] Herman, Matthew A., and Thomas Strohmer. "High-resolution radar via compressed sensing." Signal Processing, IEEE Transactions on 57.6 (2009): 2275-2284.

[6] Berger, Christian R., et al. "Application of compressive sensing to sparse channel estimation.” Communications Magazine, IEEE 48.11 (2010): 164-174.

[7] Potter, Lee C., et al. "Sparsity and compressed sensing in radar imaging." Proceedings of the IEEE 98.6 (2010): 1006-1020.

[8] Herman, Matthew, and Thomas Strohmer. "Compressed sensing radar." Radar Conference, 2008. RADAR'08. IEEE. IEEE, 2008.

[9] Bajwa, Waheed U., Kfir Gedalyahu, and Yonina C. Eldar. "Identification of parametric underspread linear systems and super-resolution radar." Signal Processing, IEEE Transactions on 59.6 (2011): 2548-2561.

[10] Gedalyahu, Kfir, and Yonina C. Eldar. "Time-delay estimation from lowrate samples: A union of subspaces approach." Signal Processing, IEEE Transactions on 58.6 (2010): 3017-3031.

[11] Sangston, Kevin J., and Karl R. Gerlach. "Coherent detection of radar targets in a non-Gaussian background." Aerospace and Electronic Systems, IEEE Transactions on 30.2 (1994): 330-340.

[12] Middleton, David. "Non-Gaussian noise models in signal processing for telecommunications: new methods an results for class A and class B noise models." Information Theory, IEEE Transactions on 45.4 (1999): 1129-1149.

[13] Ilow, Jacek, and Dimitrios Hatzinakos. "Analytic alpha-stable noise modeling in a Poisson field of interferers or scatterers." Signal Processing, IEEE Transactions on 46.6 (1998): 1601-1611.

[14] Ward, K. D., C. J. Baker, and S. Watts. "Maritime surveillance radar. I. Radar scattering from the ocean surface." Radar and Signal Processing, IEE Proceedings F. Vol. 137. No. 2. IET, 1990.

[15] Roy, Richard, and Thomas Kailath. "ESPRIT-estimation of signal parameters via rotational invariance techniques." Acoustics, Speech and Signal Processing, IEEE Transactions on 37.7 (1989): 984-995.

[16] Gill, Richard D., and Boris Y. Levit. "Applications of the van Trees inequality: a Bayesian Cramr-Rao bound.” Bernoulli (1995): 59-79. 